\title{
Signo radiológico: "Nariz del oso hormiguero"
}

Dres. Fabiola Vargas $P^{(1)}$, Karla Moënne $B^{(2)}$, Juan Escaffi $J^{(2)}$.

1. Radiólogo, Fellow Radiología Infantil, Clínica Las Condes, Universidad de Chile. Santiago - Chile.

2. Radiólogo Infantil, Departamento de Diagnóstico por Imágenes, Clínica las Condes. Santiago - Chile.

\section{Radiological sign: "The Anteater Nose"}

Abstract: The tarsal coalition corresponds to the abnormal osseous, cartilaginous or fibrous joining between two or more bones in the midfoot or hindfoot. The anteater nose sign is caused by a tubular elongation of the anterior process of the calcaneus that approaches or overlaps the tarsal scaphoid (navicular) and resembles the nose of an anteater on a lateral foot or ankle radiograph.

Keywords: Anteater nose, Tarsal coalition, Calcaneus - scaphoid.

Resumen: La coalición tarsal corresponde a la unión anormal, ya sea ósea, cartilaginosa o fibrosa, entre dos o más huesos del retro o medio pie. El signo de la nariz del oso hormiguero es causada por un alargamiento tubular del proceso anterior del calcáneo que se acerca o se superpone con el escafoides tarsiano y se asemeja a la nariz de un oso hormiguero en una radiografía lateral del pie o tobillo.

Palabras clave: Calcáneo-escafoidea, Coalición tarsal, Nariz de oso hormiguero.

Vargas F, Moënne K, Escatti J. Signo radiológico: "Nariz del oso hormiguero". Rev Chil Radiol 2014: 20(4); 156-158. Correspondencia: Fabiola Vargas P. I fabivarpe@ @otmail.com.

Trabajo recibido el 19 de marzo de 2014. Aceptado para publicación el 17 de agosto de 2014.

\section{Aspecto imaginológico}

El signo de la nariz de oso hormiguero (Figura 1) corresponde a una prolongación tubular y ensanchamiento del proceso anterior del calcáneo, visible en las radiografías laterales del pie o tobillo(1) , que se distingue de la configuración triangular normal del proceso anterior (Figura 2 a y b). Habitualmente, el proceso anterior alargado del calcáneo se superpone a la porción media del escafoides tarsiano en pacientes portadores de coalición tarsal o articulación anómala entre el proceso anterior del calcáneo y el escafoides ${ }^{(2)}$.

Aunque el signo se describe en proyecciones laterales, las coaliciones calcáneo-escafoideas tienen su mejor representación en radiografías oblicuas internas en $45^{\circ(3)}$; en esta proyección también se identifica la porción anteromedial del calcáneo anormalmente ensanchada o aplanada y la articulación anormal entre los huesos calcáneo y escafoides con superficies óseas irregulares (Figura 3).

Según un estudio retrospectivo de Crim and Kjeldsberg ${ }^{(4)}$ el signo de la nariz del oso hormiguero en las coaliciones calcáneo-escafoideas posee una sensibilidad y especificidad de $72 \%$ y $94 \%$ respectivamente, confirmando que la evaluación radiológica de rutina es una herramienta de detección valiosa para la coalición tarsal, incluso cuando se analiza por observadores inexpertos ${ }^{(4)}$.

\section{Reseña histórica}

La coalición tarsal fue descrita por primera vez por Buffon, probablemente en $1769^{(5)}$. En 1877 Zukerkandl describió por primera vez la anatomía de la coalición calcáneo-escafoidea y Kermisson fue el primero en describir el aspecto radiológico de esta condición en $1898^{(6-8)}$. En 1921 Sloman demuestró que el pie espástico peroneal está íntimamente unido a la existencia de la barra calcáneo-escafoidea y describió las características de esta coalición en la radiografía oblicua ${ }^{(6,8)}$.Posteriormente Harris y Beath (1948) relacionan también la sinostosis astrágalo-calcánea con el pie espástico peroneal.

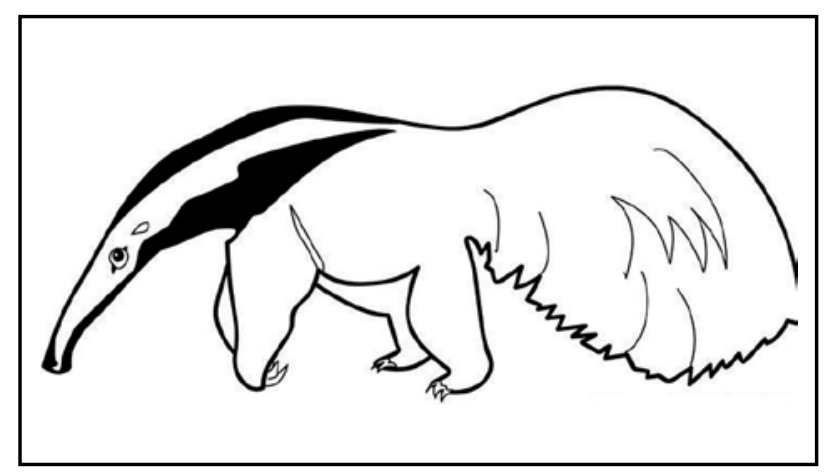

Figura 1. Esquema gráfico de un oso hormiguero. 

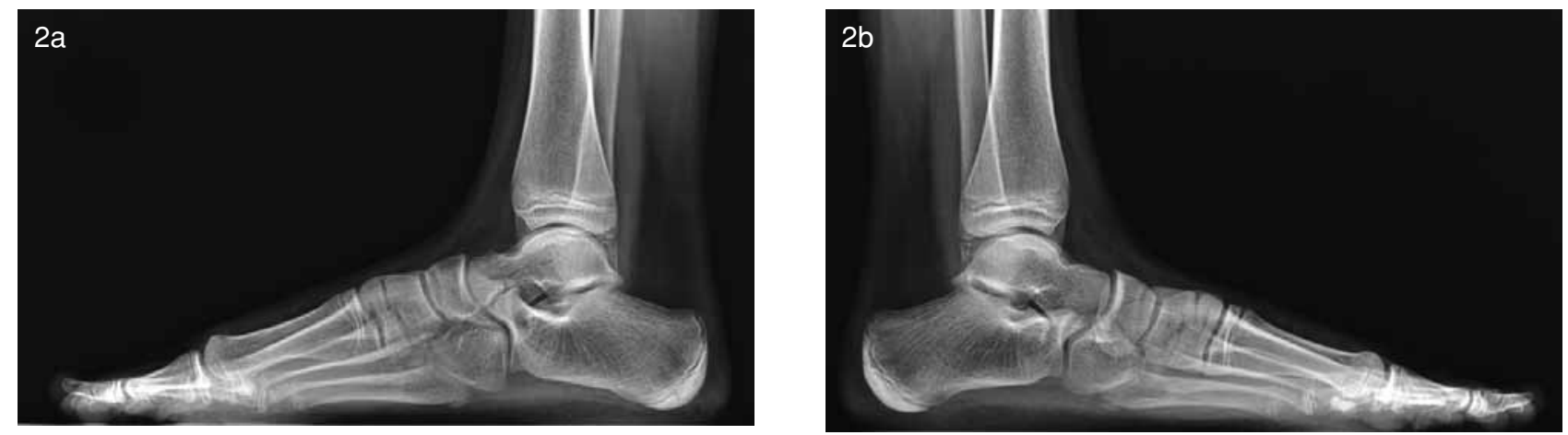

Figura 2. a) Radiografía lateral de una niña de 11 años, con coalición calcáneo- escafoidea derecha, muestra el signo "nariz de oso hormiguero". El proceso anterior del calcáneo es ancho y alargado, con punta roma b) Radiografía lateral comparativa del pie izquierdo de la misma niña, muestra el proceso anterior normal del cálcaneo con punta afilada y morfología triangular.

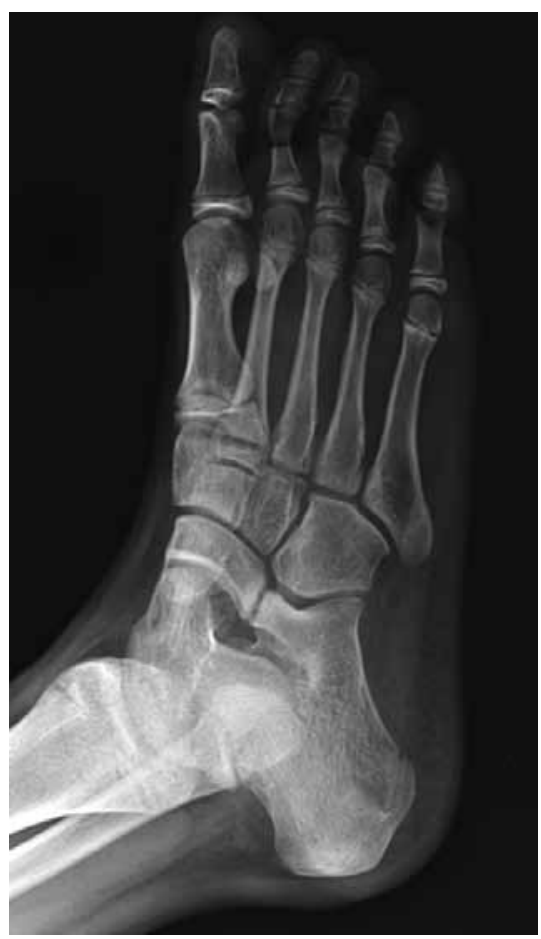

Figura 3. Coalición calcáneo escafoidea en niña de 11 años. Radiografía oblicua en 45 muestra articulación anormal entre calcáneo y escafoides tarsiano.

El signo de la nariz del oso hormiguero fue descrito por primera vez por Oestreich et. al. en $1987^{(1)}$.

Posteriormente, el descubrimiento de una variedad de coaliciones y sus caracterizaciones se han visto favorecidos por la evolución y el perfeccionamiento de la radiología simple y, más recientemente, la tomografía computarizada (TC) y la resonancia magnética (RM).

\section{Significado}

Este signo traduce la presencia de una unión anormal entre los huesos calcáneo y escafoides, debido a la falla de la segmentación anormal del mesénquima primitivo.

\section{Diagnóstico diferencial}

Los únicos falsos positivos descritos se refieren a las condiciones adquiridas que pueden simular coaliciones congénitas en los casos de enfermedades malignas, inflamaciones, fracturas intraarticulares y osteonecrosis ${ }^{(7)}$.

\section{Discusión}

La coalición tarsal es una malformación congénita infrecuente, cuyo diagnóstico es subdiagnosticado en niños y pacientes jóvenes que presentan dolor de pie y tobillo( ${ }^{(3)}$, lo que puede resultar en disminución de la movilidad, dolor y deformidad que conduce a pie plano valgo rígido ${ }^{(7)}$.

La coalición tarsal es un rasgo autosómico dominante con penetrancia variable aislada y puede ocurrir en asociación con otras anomalías ${ }^{(2)}$. Es el resultado de una diferenciación y segmentación anormal del mesénquima primitivo en el primer período del desarrollo uterino ${ }^{(3)}$, dando como resultado una fusión ósea, cartilaginosa o fibrosa anormal entre los huesos del tarso.

Las coaliciones tarsales se clasifican como: fibrosa, cartilaginosa u ósea ${ }^{(7)}$. La prevalencia se estima en $1-2 \%$ de la población ${ }^{(2-4,7)}$ y sin embargo, podría ser mayor, ya que muchos pacientes son asintomáticos 0 permanecen subdiagnosticados. Hay un ligero predominio del sexo masculino ${ }^{(3)}$ y se describen coaliciones bilaterales en alrededor del $50 \%$ de los pacientes afectados $^{(2,3,7)}$.

Las coaliciones más comunes se observan a nivel del proceso anterior del calcáneo y el escafoides tarsiano (53\%) y a nivel astrágalo-calcáneo (37\%), en este último caso, generalmente comprometiendo la faceta media ${ }^{(2-4,7)}$. La coalición talo-calcánea posterior es mucho menos común y las talo-escafoidea, calcáneo-cuboidea y cubo-escafoidea son muy poco frecuentes ${ }^{(7)}$.

Los pacientes con coalición calcáneo-escafoidea típicamente se vuelven sintomáticos entre los 8 y 12 años de edad, cuando se osifica la barra cartilaginosa calcáneo-escafoidea.

El diagnóstico clínico de la coalición no es simple y requiere examinadores expertos ${ }^{(4)}$. Los síntomas 
suelen estar presentes a fines de la etapa escolar o en la adolescencia, pero muchos de los casos se presentan en la edad adulta $^{(4)}$. Por lo general la zona de dolor es la cara lateral y antero-lateral del tobillo y frecuentemente se asocia a historia de esguince de tobillo ${ }^{(7)}$, con movimiento subtalar restringido y doloroso (eversión, inversión y deslizamiento anterior), deformidad consistente en pie plano valgo, síndrome del túnel tarsiano y espasmo del tendón peroneo ${ }^{(4)}$.

Las coaliciones fibrosas y la mayoría de las coaliciones cartilaginosas no se pueden identificar con el examen radiológico estándar y especialmente las coaliciones subastragalinas son difíciles de visualizar. Por estas razones, en la mayoría de los casos el estudio radiológico se debe complementar con TC o RM que son herramientas valiosas en la evaluación de las coaliciones tarsales ${ }^{(3)}$. Estas modalidades, además de evidenciar el signo del oso hormiguero (Figuras 4 y 5), también permiten diferenciar las coaliciones óseas de las no óseas, determinar la presencia de edema de médula ósea, extensión del compromiso articular y cambios degenerativos que afecten las articulaciones adyacentes, esto último muy importante para elegir el procedimiento terapéutico más adecuado ${ }^{(7,9)}$.

El tratamiento sintomático de la coalición calcáneoescafoidea sin cambios degenerativos de las articulaciones del tarso a menudo requiere la extirpación quirúrgica de la barra calcáneo-escafoidea, con la interposición del músculo extensor corto de los dedos ${ }^{(2)}$. Los pacientes con cambios degenerativos de las articulaciones del tarso se tratan mejor con triple artrodesis ${ }^{(2)}$.
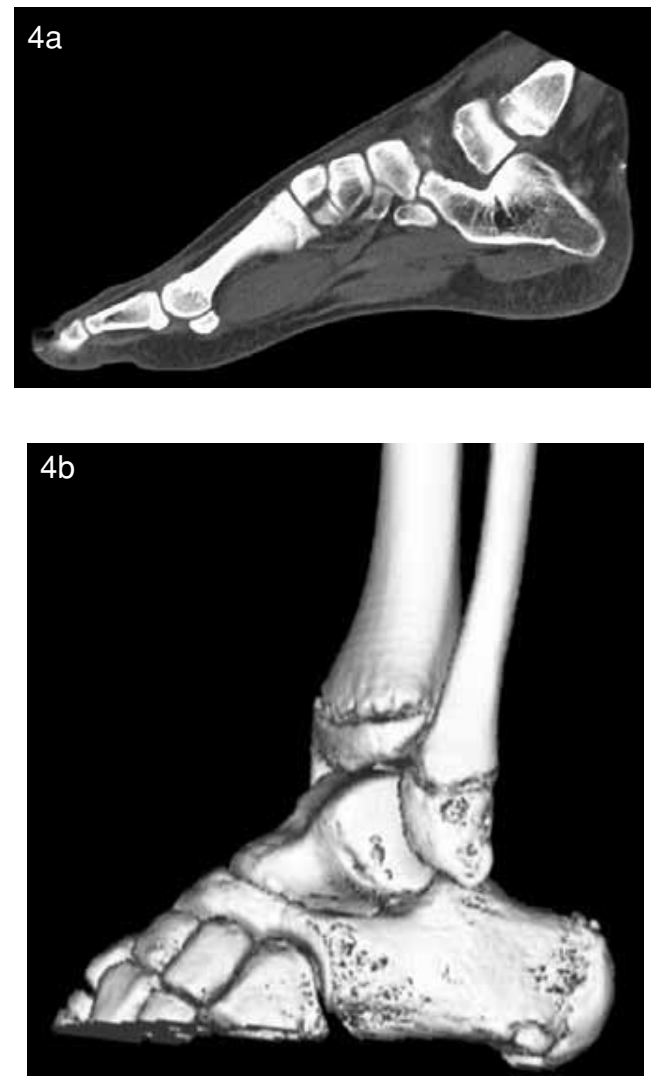

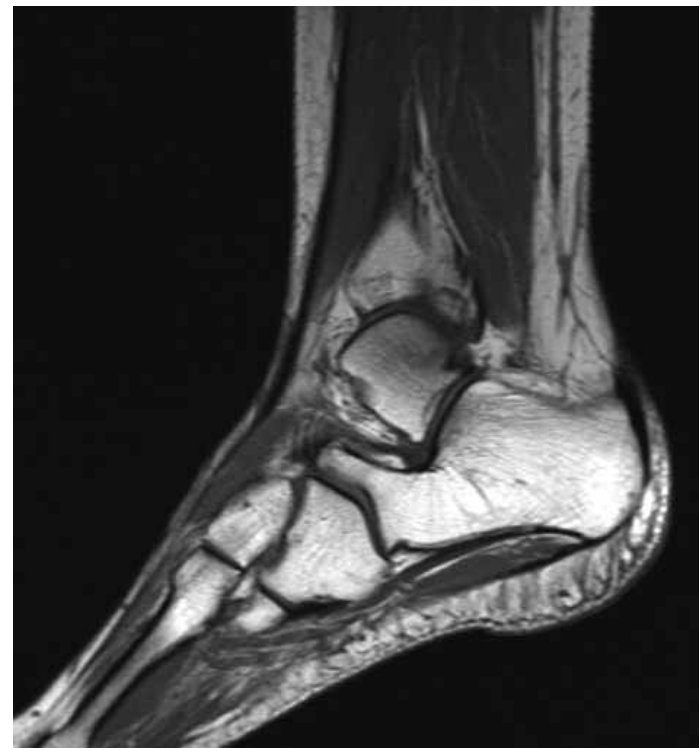

Figura 5. Aspecto de coalición calcáneo-escafoidea en $R M$, secuencia $T 1$.

\section{Conclusión}

La coalición calcáneo-escafoidea es una condición congénita que causa dolor de pies, disminución de la movilidad y deformidad que conduce a pie plano valgo rígido en niños/adolescentes, y que puede ser identificada en la mayoría de los casos con una radiografía oblicua o lateral del pie o tobillo, en la que el signo de la nariz oso hormiguero resulta de gran utilidad.

\section{Bibliografía}

1. Oestreich AE, Mize WA, Crawford AH, Morgan RC Jr. The "anteater nose": A direct sign of calcaneonavicular coalition on the lateral radiograph. J Pediatr Orthop 1987; 7(6): 709711.

2. Chapman VM. The anteater nose sign. Radiology 2007; 245(2): 604-605.

3. Newman J, Newberg A. Congenital Tarsal Coalition: Multimodality Evaluation with emphasis on CT and MR Imaging. RadioGraphics 2000; 20(2): 321-332.

4. Crim JR, Kjeldsberg KM. Radiographic diagnosis of tarsal coalition. AJR Am J Roentgenol 2004; 182(2): 323-328.

5. Sakellariou A, Claridge R. Tarsal coalition: Etiology, diagnosis and treatment. Current Orthopaedics 1998; 12(2): 135-142.

6. Espinar ES. Coaliciones tarsiales. Rev Cubana Ortop Traumatol 1996; 10(2): 138-144.

7. Efstathopoulos N, Nikolaou V, Lazarettos J, Triantopoulou Ch, Plessas S. Calcaneonavicular coalition. A case report and a literature review article. Eur J Orthop Surg Traumatol 2006; 16: 70-74.

8. Cooperman DR, Janke BE, Gilmore A, Latimer BM, Brinker MR, Thompson GH. A three-dimensional study of calcaneonavicular tarsal coalitions. J Pediatr Orthop 2001;21(5): 648-651.

9. Upasani V, Chambers RC, Mubarak SJ. Analysis of calcaneonavicular coalitions using multi-planar three-dimensional computed tomography. J Child Orthop 2008; 2(4): 301-307.

Figura 4. TC: a) Reconstrucciones planar y b) 3D, muestran coalición calcáneo escafoidea y cambios degenerativos secundarios. 